\title{
Improved Methodology for Monitoring Poly(amidoamine) Dendrimers Surface Transformations and Product Quality by Ultra Performance Liquid Chromatography
}

\author{
Chevelle A. Cason, ${ }^{1}$ Stuart A. Oehrle, ${ }^{1}$ Thomas A. Fabré, ${ }^{1}$ Craig D. Girten, ${ }^{1}$ Keith A. Walters, ${ }^{1}$ \\ Donald A. Tomalia, ${ }^{2}$ Kristi L. Haik, ${ }^{3}$ and Heather A. Bullen ${ }^{1}$ \\ ${ }^{1}$ Department of Chemistry, Northern Kentucky University, Highland Heights, KY 41099, USA \\ ${ }^{2}$ Department of Chemistry, Central Michigan University, Mt. Pleasant, MI 48859, USA \\ ${ }^{3}$ Department of Biological Sciences, Northern Kentucky University, Highland Heights, KY 41099, USA
}

Correspondence should be addressed to Heather A. Bullen, bullenh1@nku.edu

Received 7 November 2007; Revised 21 February 2008; Accepted 3 March 2008

Recommended by Donald Bansleben

Ultra performance liquid chromatography (UPLC) analysis was utilized for the first time as a methodology for monitoring poly(amidoamine) (PAMAM) dendrimer surface transformations and product quality. Results were compared to highperformance liquid chromatography (HPLC) and were found to provide a vastly improved analytical method for the characterization of dendrimer polydispersity and variance in a typical surface modification. The application of UPLC increased the average number of theoretical plates by a factor of 7 and reduced retention times of analytes by $36 \%$, while improving the resolution capability to discriminate surface variances in dendrimers. The new UPLC procedures were used to monitor surface modification of [core: ethylenediamine]; $(\mathrm{G}=4)$; dendri-poly(amidoamine)- $\left(\mathrm{NH}_{2}\right)_{64}$ (i.e., [EDA]; $(\mathrm{G} 4)$; dendri-PAMAM- $\left.\left(\mathrm{NH}_{2}\right)_{64}\right)$ to produce biotinylated dendrimer conjugates. The enhanced sensitivity and efficiency of the UPLC analyses allowed resolution of biotin substituent levels and a better characterization of the targeted dendrimer conjugates compared to traditional HPLC methodology.

Copyright $\odot 2008$ Chevelle A. Cason et al. This is an open access article distributed under the Creative Commons Attribution License, which permits unrestricted use, distribution, and reproduction in any medium, provided the original work is properly cited.

\section{INTRODUCTION}

As a subclass of dendritic polymers [1-3], dendrimers have attracted considerable attention in the past two decades largely due to the unique properties offered by this new fourth major polymer architecture [4, 5] after traditional (1) linear, (2) cross-linked (bridged), and (3) branched type categories. Dendrimers are readily produced in a wide variety of compositions as precise nanostrucures with excellent control over nanoscale sizes, shapes, nanocontainer properties, and presentation of surface chemistry $[6,7]$. As such, dendrimers are appealing to scientists as nanobuilding blocks for materials $[4,5,8]$ because of their monodisperse nanoscale sizes, guest-host encapsulation properties [9], diverse surface chemistries, and low toxicity [10]/nonimmunogenicity features [11]. Dendrimers have been utilized in a variety of nanoapplications including: gene transfection vectors [12], protein/enzyme mimicking/modeling $[13,14]$, adhesion
[15], as antiviral agents [16], sensors [17], MRI contrasting agents [18, 19], decontamination agents [20], catalysis [21], and molecular electronics [22] to mention a few. Perhaps one of the most important features of dendrimers is the ability to design mathematically defined numbers of surface terminal groups as a function of generation. These surface groups are often critical for designing polyvalent prototypes for nanopharmaceuticals $[23,24]$, nanoexcipients [25], signal amplification [26], or targeted drug delivery [27].

Such dendrimer surface modifications often create new conjugate structure complexity. Characterization of these conjugates requires analytical methodologies, which will allow a thorough understanding of important parameters such as molecular weight distribution, dendritic purity, and degree of surface functionalization. High-performance liquid chromatography (HPLC) has traditionally been utilized to separate and characterize the purity of various poly(amidoamine) PAMAM dendrimer generations or 
conjugates [28]. HPLC has also been utilized to evaluate the polydispersity, surface heterogeneity, and solubility of multifunctionalized dendrimers [29] and to study the interactions between biomolecules and dendrimers [30]. These studies as well as capillary electrophoresis (CE) [31-33] protocols have provided important characterization information for surface modified PAMAM dendrimers. However, to date, most of these chromatographic methods have proven inadequate as high-resolution characterization methodologies for monitoring the functionalization of various dendrimer surfaces. Understanding these degrees of modification is critical in designing and characterizing these nanostructures for various applications.

We wish to report the first use of ultra performance liquid chromatography (UPLC) to monitor and characterize terminal group (surface) functionalization of commercially available PAMAM $(G=4)$ dendrimers. UPLC builds on the principles of HPLC but utilizes smaller fixed phase particle sizes and elevated pressures, generating higher resolution separations of complex mixtures, increased sensitivity, and faster analysis [34, 35]. UPLC has been successfully employed for studies such as the analysis of lipids from complex biological mixtures [36] and has provided structural insights in peptides [37]. However, to our knowledge UPLC techniques have not been used to characterize dendrimers, polymers, or nanomaterials.

\section{EXPERIMENTAL METHODS}

\subsection{Materials}

Dendrimer samples; [EDA]; $(\mathrm{G}=4)$; dendri-PAMAM$\left.\left(\mathrm{NH}_{2}\right)_{64}\right)$, and [EDA]; $(\mathrm{G}=5)$; dendri-PAMAM- $\left.\left(\mathrm{NH}_{2}\right)_{128}\right)$ from standard Dendritic Nanotechnologies, Inc. (Mt. Pleasant, MI) production lots were used. All DI water was purified using a Milli-Q plus water purification system (Millipore, Bedford, MA, USA). Biotinylation of PAMAM dendrimers was accomplished using Sulfo-NHS-LC-biotin (Pierce EZ-Link Kit) [38, 39]. Briefly, dried dendrimers were resuspended in $0.1 \mathrm{M}$ phosphate buffer saline (PBS). A 3:1 molar ratio of biotin/PAMAM dendrimers was then allowed to react in $0.1 \mathrm{M}$ PBS for 2 hours on an orbital shaker [38, 39]. Excess, unreacted biotin was then removed using Microcon filters (Millipore. Bedford, MA, USA). Biotinylated dendrimer conjugates were subsequently rinsed with PBS followed by DI water. For chromatographic analysis, all dendrimer samples were dried and redissolved in the HPLC mobile phase, which consisted of a 90:10 mixture of DI water/acetonitrile (ACN) with $0.14 \%$ trifluoroacetic acid (TFA).

\subsection{High-performance liquid chromatography}

A reversed phase HPLC system consisting of a Waters Breeze HPLC (Waters Corporation, Milford, MA USA), equipped with a 717 plus autosampler, 2487 dual $\lambda$ UV detector, $5 \mu \mathrm{m}$ Symmetry $300 \mathrm{C} 18$ column $(4.6 \mathrm{~mm} \times 150 \mathrm{~mm})$, and a Waters Sentry Symmetry C18 guard column was used. The mobile phase consisted of a linear gradient beginning with
$90 \%$ water and $10 \% \mathrm{ACN}$ (each with $0.14 \%$ TFA) at a flow rate of $1 \mathrm{~mL} / \mathrm{min}$ reaching $68 \%$ water $32 \% \mathrm{ACN}$ over 10 minutes. The injection volume was $100 \mu \mathrm{L}$, and detection of eluted samples was performed at $214 \mathrm{~nm}$. These parameters were chosen based on optimization of previous reported HPLC methods of dendrimers [28, 40-42].

\subsection{Ultra performance liquid chromatography}

UPLC analyses were performed with a Waters Acquity Ultra Performance LC system (Waters Corporation, Milford, MA, USA). UPLC separation was achieved on an Acquity UPLC BEH C18 column $(100 \mathrm{~mm} \times 2.1 \mathrm{~mm}$, i.d., $1.7 \mu \mathrm{m}$ particle size, $130 \AA$ pore size, Waters) maintained at $40^{\circ} \mathrm{C}$. The compounds were injected using a partial loop injection of $7 \mu \mathrm{L}$. Detection was done using the UPLC photodiode array detector monitoring at $214 \mathrm{~nm}$. The previous HPLC method was scaled geometrically for the UPLC system for comparison using a conversion software program that scales all parameters of the method to the UPLC column [43]. The mobile phase consisted of $0.02 \%$ TFA in water (solvent A) and $0.018 \%$ TFA in ACN (solvent B). The gradient used for the UPLC method consisted of linear gradient from $99 \%$ solvent A to $65 \%$ solvent A over 14 minutes followed by a 2-minute hold and back to initial conditions. A flow rate of $0.4 \mathrm{~mL} / \mathrm{min}$ was used.

\section{RESULTS AND DISCUSSION}

HPLC can be used to evaluate the purity and distribution of various dendrimer generations. Figure 1 shows the HPLC analysis of G4 amine terminated and G4 biotinylated PAMAMs. A chromatogram of G5; amine terminated PAMAM is also included for comparison. Amine terminated PAMAMs tend to readily adsorb to a variety of substrates, therefore reverse ion pair chromatography was conducted using TFA [29, 44]. Figure 1 clearly illustrates an enhancement in retention time as a function of PAMAM generation, due to the geometrically increased surface density of the terminal amine groups and TFA ion pairs. Results for G4 and G5 are similar to those reported previously [28]. While PAMAM dendrimer populations are relatively monodisperse compared to traditional polymer architectures, they still exhibit minor defect levels, which may be described as generational or skeletal dispersity (i.e., missing arms and/or intramolecular loops) $[4,5,30]$. The polydispersity of the PAMAM dendrimers can be estimated qualitatively by assessing the peak width at half height $\left(\mathrm{W}_{\mathrm{H} / 2}\right)$ [28]. Results suggest that generational defect levels increase with dendrimer generation (e.g., G4, PAMAM, $\mathrm{W}_{\mathrm{H} / 2} / \mathrm{min}=0.39$, whereas, G5; PAMAM, $\left.\mathrm{W}_{\mathrm{H} / 2} / \mathrm{min}=0.49\right)$. These trends in $\mathrm{W}_{\mathrm{H} / 2}$ persisted regardless of changes in HPLC operating conditions. Both G4 and G5, amine terminated PAMAM generations exhibited a small shoulder to the right of the main band, which closely overlaps with the next higher generation peak (see Figure 1). This indicates the presence of dimers $(<10 \%$ of sample), which is in agreement with previous reports in the literature [31, 45-47]. It should also be noted that unlike earlier reports $[28,30]$, traces of trailing 


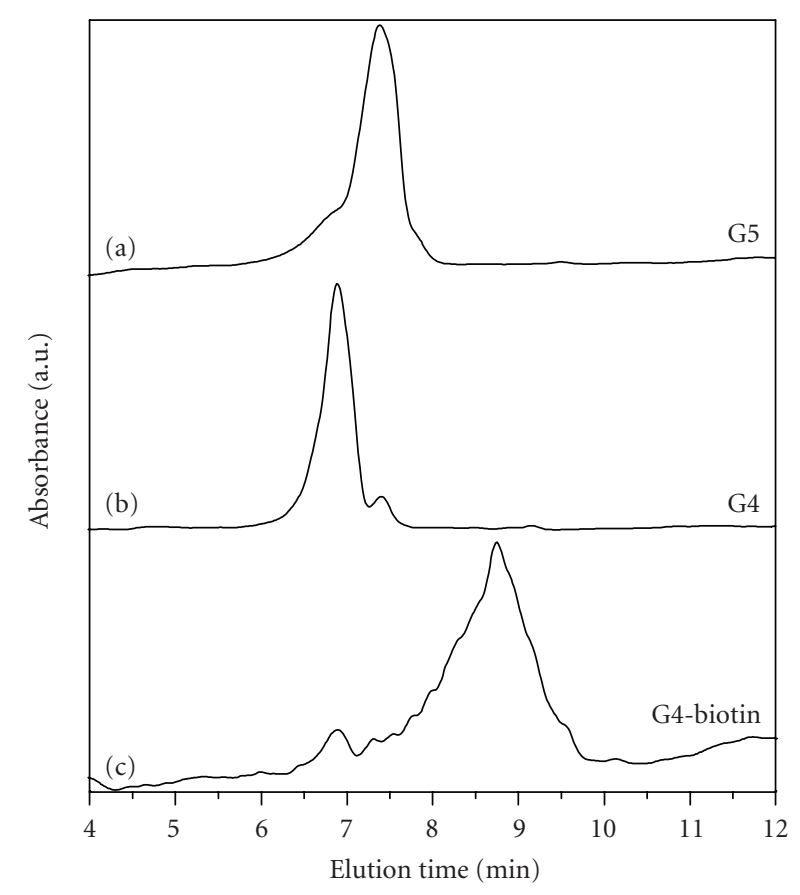

FIgURE 1: HPLC chromatogram of (a) [EDA core], $G=5$, amine terminated PAMAM dendrimers, (b) [EDA core], $\mathrm{G}=4$, amine terminated PAMAM dendrimers, and (c) [EDA core], $\mathrm{G}=4$, biotinylated PAMAM dendrimers. The concentrations of each dendrimer in solution are $0.75 \mathrm{mg} / \mathrm{mL}$.

lower generation PAMAMs are not apparent with the G4, PAMAM, however they are evident with the G5, PAMAM. The G4, biotinylated PAMAM dendrimers, exhibited broader elution profiles $\left(\mathrm{W}_{\mathrm{H} / 2} / \mathrm{min}=1.05\right)$ with evidence of band overlap (see Figure 1). This elution peak profile suggests that there are varied degrees of surface functionalization, which remain unresolved using HPLC. The chromatogram also indicates the presence of $\sim 6 \%$ unreacted PAMAM dendrimers for the synthesis of G4-biotinylated conjugates.

Figure 2 illustrates a UPLC analysis of the same G4; amine terminated PAMAM dendrimer and biotinylated conjugate. It appears that the main elution peak associated with G4, amine terminated PAMAM (see Figure 2(a)), consists of a series of at least 5 differentiated overlapping bands. The dimer peak is also present along with impurities associated with TFA (6.26, 6.87, and 7.47 minutes) and an unidentifiable impurity at 5.66 minutes. At this time, the impurity is unknown and is absent in the analysis of biotinylated conjugates and all HPLC analyses.

The UPLC chromatogram of G4 PAMAMs, Figure 2(a), provides insight into the polydispersity of higher generation PAMAMs. These variations (or defects) are typically associated with the successive iterations of divergent synthesis $[5,48]$. The sensitivity for chromatographically separating structural dispersities, such as dimers and trailing generations, decreases with higher generations of PAMAMs. Researchers have also suggested that these components along with minor skeletal defects are likely masked inside the main chromatographic peak [28, 45, 47, 49]. Results

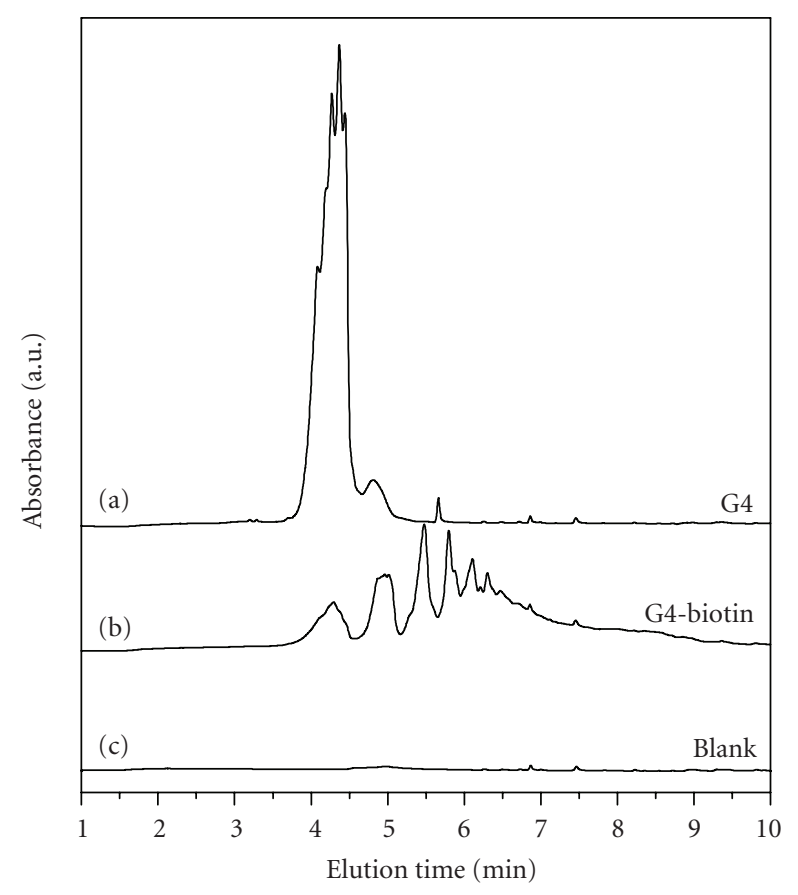

FIgURE 2: UPLC chromatogram of (a) [EDA core], $G=4$, amine terminated PAMAM dendrimers, (b) [EDA core], $\mathrm{G}=$ 4, biotinylated PAMAMs and, (c) control blank of TFA. The concentrations of each dendrimer in solution are $0.50 \mathrm{mg} / \mathrm{mL}$.

here provide details for the first time into the skeletal differentiation of PAMAMs. Although, UPLC relies on the same selectivity and retention methods as HPLC, the smaller particle sizes in UPLC enable improved limits in the chromatographic separation [35]. This is evident by the presence of multiple bands within the main peak associated with G4 (see Figure 2(a)). Furthermore, the UPLC analysis of biotinylated G4 PAMAMs demonstrates the advantage gained by using smaller particle sizes and higher pressures to evaluate the substitutional diversity of dendrimers upon surface modification (see Figure 2(b)).

A comparison of HPLC and UPLC analyses of G4 bioinylated PAMAMs is shown in Figure 3. In both analyses, a peak is present to the left of the main band, associated with unreacted G4 PAMAM. The analyses indicate that $\sim 6 \%$ of the G4 PAMAMs were unmodified. The focus of this work, however, is to demonstrate the new resolution capabilities to discriminate surface variances that are possible while monitoring the synthesis of various biotinylated-PAMAM conjugates. The UPLC chromatogram for biotinylated G4, PAMAMs (see Figure 3(b)), demonstrates an improved separation/resolution of biotinylated PAMAM conjugates compared to the HPLC analysis, as seen in Figure 3(a). Apart from the G4, PAMAM peak, Figure 3(b) exhibits 6 major peaks $\left(\mathrm{t}_{\mathrm{r} 1}=4.98 \mathrm{~min}, \mathrm{t}_{\mathrm{r} 2}=5.48 \mathrm{~min}, \mathrm{t}_{\mathrm{r} 3}=5.80, \mathrm{t}_{\mathrm{r} 4}=6.11 \mathrm{~min}\right.$, $\left.\mathrm{t}_{\mathrm{r} 5}=6.30, \mathrm{t}_{\mathrm{r} 6}=6.47\right)$, associated with varied degrees of surface functionalization. NMR analysis (not shown here) indicates $\sim 17 \%$ surface coverage. However, UPLC analysis reveals that there are significant variations in the overall 
TABLE 1: Comparison of HPLC and UPLC analyses of [EDA core] $\mathrm{G}=4 ; \mathrm{NH}_{2}$ and $\mathrm{G}=4$; biotinylated PAMAM dendrimers. (*Indicates dimer, ${ }^{\dagger}$ indicates unfunctionalized $\left(\mathrm{G}=4 ; \mathrm{NH}_{2}\right)$ ).

\begin{tabular}{|c|c|c|c|c|}
\hline Sample & Method & Retention time, $\mathrm{t}_{\mathrm{r}}(\mathrm{min})$ & $\mathrm{W}_{\mathrm{H} / 2}(\min )$ & Theoretical Plates $(\mathrm{N})$ \\
\hline \multirow{8}{*}{$\mathrm{G}=4 ; \mathrm{NH}_{2}$} & \multirow{2}{*}{ HPLC } & 6.90 & 0.39 & 1737 \\
\hline & & $7.41^{*}$ & 0.24 & 5290 \\
\hline & \multirow{6}{*}{ UPLC } & 4.08 & 0.10 & 9239 \\
\hline & & 4.21 & 0.07 & 20075 \\
\hline & & 4.27 & 0.07 & 20651 \\
\hline & & 4.37 & 0.07 & 21630 \\
\hline & & 4.44 & 0.09 & 13507 \\
\hline & & $4.81^{*}$ & 0.26 & 1899 \\
\hline \multirow{9}{*}{$\mathrm{G}=4$; biotinylated } & \multirow{2}{*}{ HPLC } & $6.90^{\dagger}$ & 0.40 & 1651 \\
\hline & & 8.75 & 1.05 & 385 \\
\hline & \multirow{7}{*}{ UPLC } & $4.30^{\dagger}$ & 0.41 & 610 \\
\hline & & 4.98 & 0.28 & 1755 \\
\hline & & 5.48 & 0.14 & 8503 \\
\hline & & 5.80 & 0.13 & 11047 \\
\hline & & 6.11 & 0.20 & 5180 \\
\hline & & 6.30 & 0.14 & 11238 \\
\hline & & 6.47 & 0.17 & 8039 \\
\hline
\end{tabular}

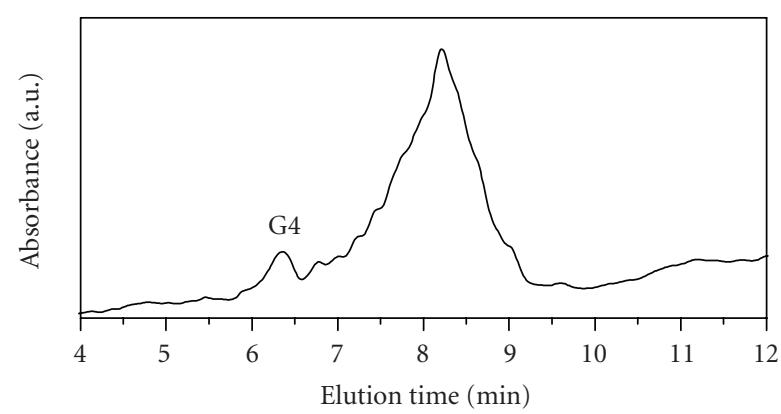

(a)

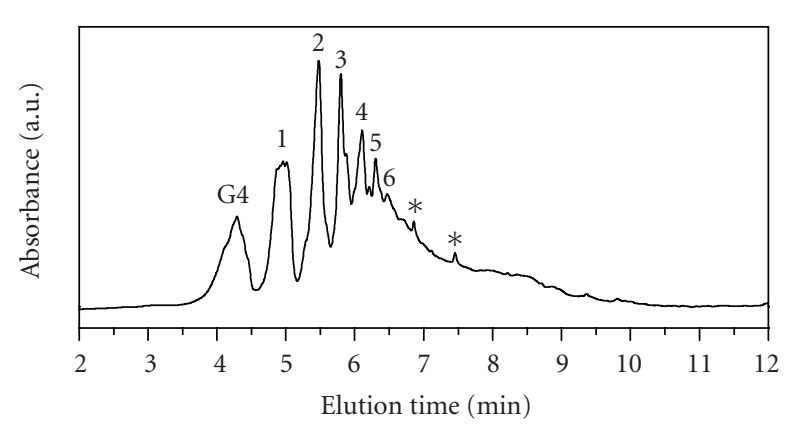

(b)

FIGURE 3: Comparison of (a) HPLC and (b) UPLC analyses of [EDA core]; G = 4 biotinylated PAMAMs. The solution concentrations of each dendrimer in solution are $0.50 \mathrm{mg} / \mathrm{mL}$ (*indicates TFA contaminate).

degree of surface modification. If a Gaussian distribution of surface functionalization is assumed, the present results suggest, based on peak areas, that fractions 3,4 , and 5 contain dendrimer conjugates with larger biotin coverage, and fractions 1 and 2 contain biotinylated conjugates with a lower degree of functionalization.

These results illustrate the enhanced potential of UPLC to assess generational defect levels as well as degree of dendrimer surface modification in greater detail than has been possible by HPLC analyses or other chromatographic methods such as CE $[31-33,50]$. Current methods utilized to determine the degree of functionality such as NMR and matrix assisted laser desorption/ionization-time of flight (MALDI-TOF) only provide an estimated average number of surface functional moieties. However, if UPLC could be coupled with ESI-TOF or MALDI-TOF, analysis of the more highly resolved/separated fractions should provide deeper insights into the species identification of surface modified dendrimer conjugates.
Furthermore, UPLC allows for increased efficiency and sensitivity in analysis. This phenomena may be explained in accordance with the van Deemter equation [51]; wherein, smaller particles provide not only increased efficiency, but also the ability to work at increased linear velocity without a loss of efficiency, thus providing both resolution and speed. A summary of the separation comparison can be found in Table 1. For example, utilizing the scaled HPLC separation method, the retention time for G4 PAMAMs is decreased from 6.90 minutes to 4.30 minutes, reducing the run time required for characterizing $\mathrm{G}=4$, PAMAM dendrimers by $36 \%$. It should be noted that this reduction in run time could potentially be improved with further modification of the UPLC method. UPLC analysis also produces a 7-fold increase in average number of theoretical plates, improving the capability to distinguish dendrimer surface variances. In addition, due to the reduced band spreading during the separation process, the analytes are more concentrated at the point of detection. This allows for lower injection 
volumes and concentrations of analyte, improving the limit of detection of by a factor of 100 (HPLC method for G4, PAMAMs, is $1.6 \times 10^{-10} \mathrm{moL}$ compared to $1.6 \times 10^{-12} \mathrm{moL}$ for UPLC).

\section{CONCLUSIONS}

UPLC provides a significantly more detailed analysis of PAMAM dendrimer generational defect levels. These PAMAMs have been widely used in diagnostics [52], drug delivery [25], and gene transfection $[53,54]$ types of commercial applications. Furthermore, this new methodology has provided more resolved elution profiles for biotin surface functionalized dendrimers. The findings from this present study indicate that UPLC is an improved chromatographic method for the detection, purification, and separation of unmodified as well as surface modified PAMAM dendrimers. This characterization method should be invaluable for the characterization of a wide variety of surface modified dendrimers, which are known to be critical in many emerging commercial applications $[6,55]$.

\section{ACKNOWLEDGMENTS}

This project has been funded by the Merck Institute for Science Education, Kentucky NSF EPSCoR, Northern Kentucky University, Center for Integrated Natural Science and Mathematics, and the NKU Research Foundation. Infrastructure support was provided, in part, by the National Center for Research Resources Grant P20 RR16481. The authors would also like to thank Waters Corporation for continued support of research at Northern Kentucky University.

\section{REFERENCES}

[1] D. A. Tomalia and J. M. J. Fréchet, "Discovery of dendrimers and dendritic polymers: a brief historical perspective," Journal of Polymer Science Part A, vol. 40, no. 16, pp. 2719-2728, 2002.

[2] D. A. Tomalia and J. M. J. Fréchet, Dendrimers and other Dendritic Polymers, John Wiley \& Sons, Chichester, UK, 2001.

[3] D. A. Tomalia, "The emergence of a new macromolecular architecture: "the dendritic state", in Physical Properties of Polymers Handbook, J. E. Mark, Ed., pp. 671-692, Springer, New York, NY, USA, 2007.

[4] D. A. Tomalia, S. Uppuluri, D. R. Swanson, and J. Li, "Dendrimers as reactive modules for the synthesis of new structure-controlled, higher-complexity megamers," Pure and Applied Chemistry, vol. 72, no. 12, pp. 2343-2358, 2000.

[5] D. A. Tomalia, "Birth of a new macromolecular architecture: dendrimers as quantized building blocks for nanoscale synthetic organic chemistry," Progress in Polymer Science, vol. 30, no. 3-4, pp. 294-324, 2005.

[6] D. A. Tomalia, "The dendritic state," Materials Today, vol. 8, no. 3, pp. 34-46, 2005.

[7] D. R. Swanson, B. Huang, H. G. Abdelhady, and D. A. Tomalia, "Unique steric and geometry induced stoichiometries observed in the divergent synthesis of poly(esteracrylate/amine) (PEA) dendrimers," New Journal of Chemistry, vol. 31, no. 7, pp. 1368-1378, 2007.
[8] D. A. Tomalia, S. A. Henderson, and M. S. Diallo, "Dendrimers-an enabling synthetic science to controlled organic nanostructures," in Handbook of Nanoscience, Engineering and Technology, W. A. I. Goddard, D. W. Brenner, S. E. Lyshevski, and G. J. Iafrate, Eds., pp. 24.1-24.47, CRC Press, Taylor and Francis, Boca Raton, Fla, USA, 2007.

[9] R. Haag, "Dendrimers and hyperbranched polymers as highloading supports for organic synthesis," Chemistry: A European Journal, vol. 7, no. 2, pp. 327-335, 2001.

[10] M. Najlah, S. Freeman, A. David, and A. D'Emanuele, “Synthesis and assessment of first-generation polyamidoamine denrimer prodrugs to enhance the cellular permeability of Pgp Substrates," Bioconjugate Chemistry, vol. 18, no. 3, pp. 937946, 2007.

[11] H. Kobayashi, S. Kawamoto, T. Saga, et al., "Positive effects of polyethylene glycol conjugation to generation-4 polyamidoamine dendrimers as macromolecular MR contrast agents," Magnetic Resonance in Medicine, vol. 46, no. 4, pp. 781-788, 2001.

[12] D. R. Radu, C.-Y. Lai, K. Jeftinija, E. W. Rowe, S. Jeftinija, and V. S.-Y. Lin, "A polyamidoamine dendrimer-capped mesoporous silica nanosphere-based gene transfection reagent," Journal of the American Chemical Society, vol. 126, no. 41, pp. 13216-13217, 2004.

[13] D. A. Tomalia, B. Huang, D. R. Swanson, H. M. Brothers II, and J. W. Klimash, "Structure control within poly(amidoamine) dendrimers: size, shape and regiochemical mimicry of globular proteins," Tetrahedron, vol. 59, no. 22, pp. 3799-3813, 2003.

[14] J. Kofoed and J.-L. Reymond, "Dendrimers as artificial enzymes," Current Opinion in Chemical Biology, vol. 9, no. 6, pp. 656-664, 2005.

[15] X. Li, F. Huang, M. Curry, S. C. Street, and M. L. Weaver, "Improved adhesion of Au thin films to $\mathrm{SiOx} / \mathrm{Si}$ substrates by dendrimer mediation," Thin Solid Films, vol. 473, no. 1, pp. 164-168, 2005.

[16] D. L. Patton, Y. T. Cosgrove Sweeney, T. D. McCarthy, and S. L. Hillier, "Preclinical safety and efficacy assessments of dendrimer-based (sp17013) microbicide gel formulations in nonhuman primate model," Antimicrobial Agents and Chemotherapy, vol. 50, no. 5, pp. 1696-1700, 2006.

[17] J. Losada, M. Zamora, P. G. Armada, I. Cuadrado, B. Alonso, and C. M. Casado, "Bioenzyme sensors based on novel polymethylferrocenyl dendrimers," Analytical and Bioanalytical Chemistry, vol. 385, no. 7, pp. 1209-1217, 2006.

[18] J. W. Dear, H. Kobayashi, M. W. Brechbiel, and R. A. Star, "Imaging acute renal failure with polyamine dendrimer-based MRI contrast agents," Nephron Clinical Practice, vol. 103, no. 2, pp. c45-c49, 2006.

[19] H. Kobayashi and M. W. Brechbiel, "Dendrimer-based nanosized MRI contrast agents," Current Pharmaceutical Biotechnology, vol. 5, no. 6, pp. 539-549, 2004.

[20] M. S. Diallo, S. Christie, P. Swaminathan, J. H. Johnson Jr., and W. A. Goddard III, "Dendrimer enhanced ultrafiltration. 1. Recovery of $\mathrm{Cu}$ (II) from aqueous solutions using PAMAM dendrimers with ethylene diamine core and terminal $\mathrm{NH}_{2}$ groups," Environmental Science \& Technology, vol. 39, no. 5, pp. 1366-1377, 2005.

[21] J. N. H. Reek, D. de Groot, G. E. Oosterom, P. C. J. Kamer, and P. W. N. M. van Leeuwen, "Core and periphery functionalized dendrimers for transition metal catalysis; a covalent and a non-covalent approach," Journal of Biotechnology, vol. 90, no. 3-4, pp. 159-181, 2002. 
[22] J. M. Lupton, I. D. W. Samuel, P. L. Burn, and S. Mukamel, "Exciton confinement in organic dendrimer quantum wells for opto-electronic applications," Journal of Chemical Physics, vol. 116, no. 2, pp. 455-459, 2002.

[23] Y.-H. Jiang, P. Emau, J. S. Cairns, et al., "SPL7013 gel as a topical microbicide for prevention of vaginal transmission of SHIV $_{89.6 \mathrm{p}}$ in Macaques," AIDS Research and Human Retroviruses, vol. 21, no. 3, pp. 207-213, 2005.

[24] S. Fuchs, T. Kapp, H. Otto, et al., "A surface-modified dendrimer set for potential application as drug delivery vehicles: synthesis, in vitro toxicity, and intracellular localization," Chemistry: A European Journal, vol. 10, no. 5, pp. 1167-1192, 2004.

[25] S. Svenson and D. A. Tomalia, "Dendrimers in biomedical applications - reflections on the field," Advanced Drug Delivery Reviews, vol. 57, no. 15, pp. 2106-2129, 2005.

[26] H. Xu, C. A. S. Regino, M. Bernardo, et al., "Toward improved syntheses of dendrimer-based magnetic resonance imaging contrast agents: new bifunctional diethylenetriaminepentaacetic acid ligands and non aqueous conjugation chemistry," Journal of Medicinal Chemistry, vol. 50, no. 14, pp. 3185-3193, 2007.

[27] D. A. Tomalia, L. A. Reyna, and S. Svenson, "Dendrimers as multi-purpose nanodevices for oncology drug delivery and diagnostic imaging," Biochemical Society Transactions, vol. 35, no. 1, pp. 61-67, 2007.

[28] M. T. Islam, X. Shi, L. Balogh, and J. R. Baker Jr., "HPLC separation of different generations of poly(amidoamine) dendrimers modified with various terminal groups," Analytical Chemistry, vol. 77, no. 7, pp. 2063-2070, 2005.

[29] M. T. Islam, I. J. Majoros, and J. R. Baker Jr., "HPLC analysis of PAMAM dendrimer based multifunctional devices," Journal of Chromatography B, vol. 822, no. 1-2, pp. 21-26, 2005.

[30] X. Shi, X. Bi, T. R. Ganser, et al., "HPLC analysis of functionalized poly(amidoamine) dendrimers and the interaction between a folate-dendrimer conjugate and folate binding protein," Analyst, vol. 131, no. 7, pp. 842-848, 2006.

[31] X. Shi, I. J. Majoros, and J. R. Baker Jr., "Capillary electrophoresis of poly(amidoamine) dendrimers: from simple derivatives to complex multifunctional medical nanodevices," Molecular Pharmaceutics, vol. 2, no. 4, pp. 278-294, 2005.

[32] X. Shi, I. Bányai, M. T. Islam, et al., "Generational, skeletal and substitutional diversities in generation one poly(amidoamine) dendrimers," Polymer, vol. 46, no. 9, pp. 3022-3034, 2005.

[33] X. Shi, I. Bányai, K. Rodriguez, et al., "Electrophoretic mobility and molecular distribution studies of poly(amidoamine)dendrimers of defined charges," Electrophoresis, vol. 27, no. 9, pp. 1758-1767, 2006.

[34] I. D. Wilson, J. K. Nicholson, J. Castro-Perez, et al., "High resolution "ultra performance" liquid chromatography coupled to oa-TOF mass spectrometry as a tool for differential metabolic pathway profiling in functional genomic studies," Journal of Proteome Research, vol. 4, no. 2, pp. 591-598, 2005.

[35] M. E. Swartz, "UPLC ${ }^{\mathrm{TM}}$ : an introduction and review," Journal of Liquid Chromatography \& Related Technologies, vol. 28, no. 7-8, pp. 1253-1263, 2005.

[36] P. D. Rainville, C. L. Stumpf, J. P. Shockcor, R. S. Plumb, and J. K. Nicholson, "Novel application of reversed-phase UPLCoaTOF-MS for lipid analysis in complex biological mixtures: a new tool for lipidomics," Journal of Proteome Research, vol. 6, no. 2, pp. 552-558, 2007.

[37] M. Cindrić, T. Čepo, N. Galić, et al., "Structural characterization of PEGylated $\mathrm{rHuG-CF}$ and location of PEG attachment sites," Journal of Pharmaceutical and Biomedical Analysis, vol. 44, no. 2, pp. 388-395, 2007.

[38] T. A. Fabré, C. A. Cason, J. D. Hogan, et al.,in preparation.

[39] Z. Liu and M. D. Amiridis, "FT-IRRAS spectroscopic studies of the interaction of avidin with biotinylated dendrimer surfaces," Colloids and Surfaces B, vol. 35, no. 3-4, pp. 197-203, 2004.

[40] A. D’Emanuele, R. Jevprasesphant, J. Penny, and D. Attwood, "The use of a dendrimer-propranolol prodrug to bypass efflux transporters and enhance oral bioavailability," Journal of Controlled Release, vol. 95, no. 3, pp. 447-453, 2004.

[41] B. Devarakonda and M. M. de Villiers, "Development and validation of an HPLC method involving solid-phase extraction for the analysis of hydrophobic drugs in the presence of polyamidoamine (PAMAM) dendrimers," Journal of Liquid Chromatography and Related Technologies, vol. 28, no. 15, pp. 2325-2338, 2005.

[42] A. Clouet, T. Darbre, and J.-L. Reymond, "Combinatorial synthesis, selection, and properties of esterase peptide dendrimers," Biopolymers, vol. 84, no. 1, pp. 114-123, 2006.

[43] E. Grumbach, T. Wheat, D. McCabe, D. Diehl, and J. Mazzeo, "Improving LC separations: transferring methods from HPLC to UPLC ${ }^{\mathrm{TM}}$," LC/GC North America, vol. 24, pp. 80-81, 2006.

[44] Y. Chen, A. R. Mehok, C. T. Mant, and R. S. Hodges, "Optimum concentration of trifluoroacetic acid for reversedphase liquid chromatography of peptides revisited," Journal of Chromatography A, vol. 1043, no. 1, pp. 9-18, 2004.

[45] A. Ebber, M. Vaher, J. Peterson, and M. Lopp, "Application of capillary zone electrophoresis to the separation and characterization of poly(amidoamine) dendrimers with an ethylenediamine core," Journal of Chromatography A, vol. 949, no. 1-2, pp. 351-358, 2002.

[46] J. Peterson, A. Abber, V. Allikmaa, and M. Lopp, "Synthesis and CZE analysis of PAMAM dendrimers with an ethylenediamine core," Proceedings of the Estonian Academy of Sciences. Chemistry, vol. 50, pp. 156-166, 2001.

[47] P. Sedláková, J. Svobodová, I. Mikšík, and H. Tomás, “Separation of poly(amidoamine) (PAMAM) dendrimer generations by dynamic coating capillary electrophoresis," Journal of Chromatography B, vol. 841, no. 1-2, pp. 135-139, 2006.

[48] G. J. Kallos, S. Lewis, J. Zhou, D. M. Hedstrand, and D. A. Tomalia, "Molecular weight determination of a polyamidoamine starburst polymer by electrospray-ionization mass spectrometry," Rapid Communications in Mass Spectrometry, vol. 5, no. 9, pp. 383-386, 1991.

[49] J. Peterson, V. Allikmaa, J. Subbi, T. Pehk, and M. Lopp, "Structural deviations in poly(amidoamine) dendrimers:a MALDI-TOF MS analysis," European Polymer Journal, vol. 39, no. 1, pp. 33-42, 2003.

[50] A. Desai, X. Shi, and J. R. Baker Jr., "CE of poly(amidoamine) succinamic acid dendrimers using a poly(vinyl alcohol)coated capillary," Electrophoresis, vol. 29, no. 2, pp. 510-515, 2008.

[51] J. J. van Deemter, F. J. Zuiderweg, and A. Klinkenberg, "Longitudinal diffusion and resistance to mass transfer as causes of nonideality in chromatography," Chemical Engineering Science, vol. 5, no. 6, pp. 271-289, 1956.

[52] P. Singh, "Dendrimer-based biological reagents: preparation and applications in diagnostics," in Dendrimers and other Dendritic Polymers, D. A. Tomalia and J. M. J. Fréchet, Eds., pp. 463-484, John Wiley \& Sons, Chichester, UK, 2001. 
[53] L. A. Kubasiak and D. A. Tomalia, "Cationic dendrimers as gene transfection vectors: dendri-poly(amidoamines) and dendri-poly(propylenimines)," in Polymeric Gene Delivery: Principles and Applications, M. M. Amiji, Ed., pp. 133-157, CRC Press, Boca Raton, Fla, USA, 2004.

[54] C. S. Braun, J. A. Vetro, D. A. Tomalia, G. S. Koe, J. G. Koe, and C. R. Middaugh, "Structure/function relationships of polyamidoamine/DNA dendrimers as gene delivery vehicles," Journal of Pharmaceutical Sciences, vol. 94, no. 2, pp. 423-436, 2005.

[55] D. A. Tomalia, "Dendrons/dendrimers. The convergence of quantized dendridic building blocks/architectures for applications in nanotechnology," Chemistry Today, vol. 23, no. 6, pp. 41-45, 2005. 

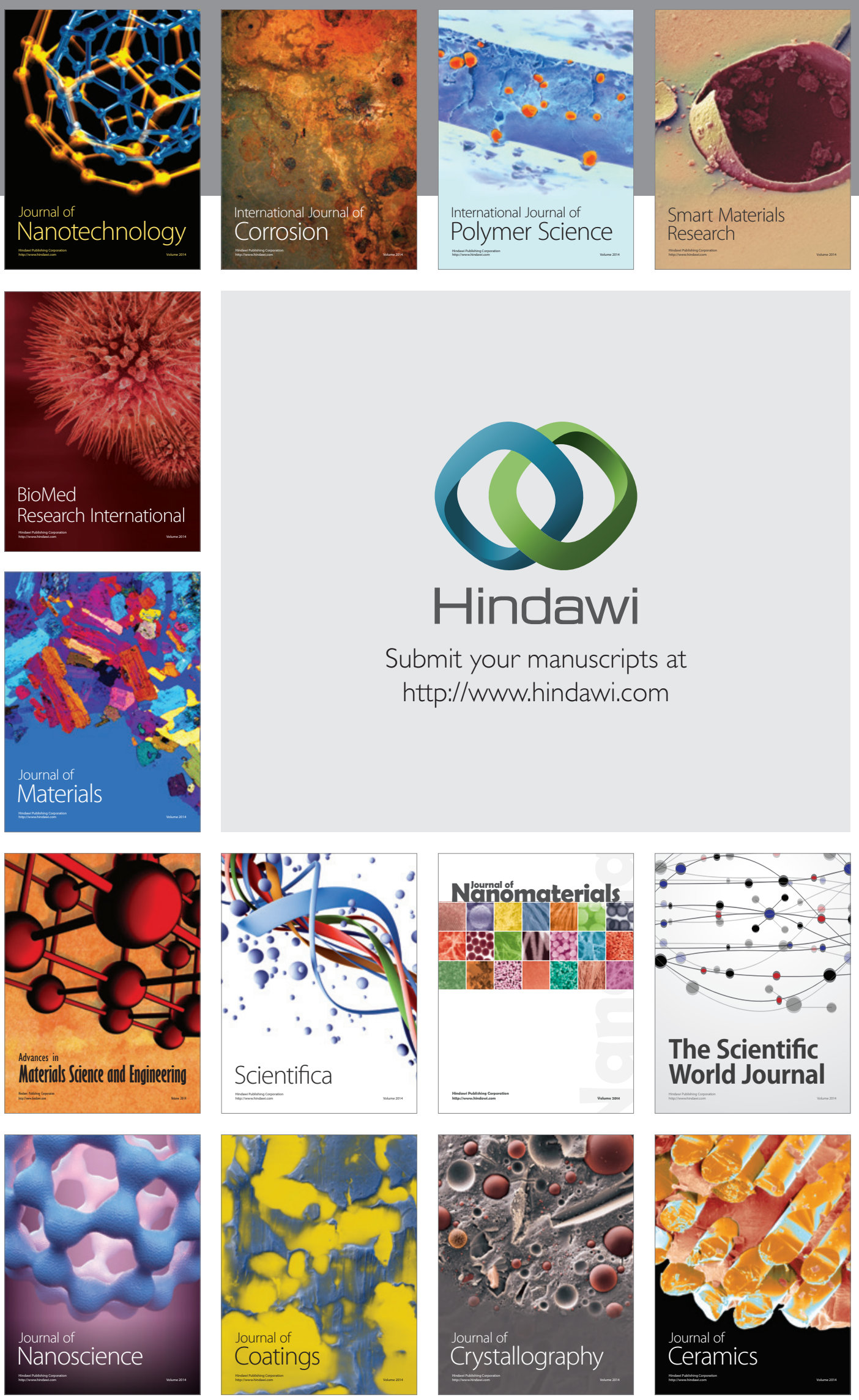

The Scientific World Journal

Submit your manuscripts at

http://www.hindawi.com

\section{World Journal}

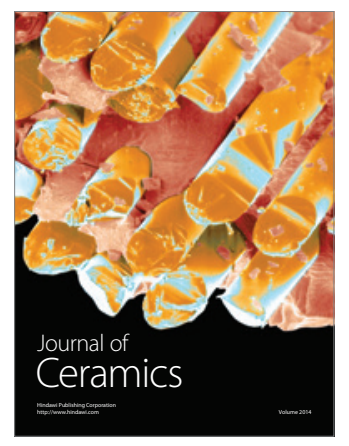

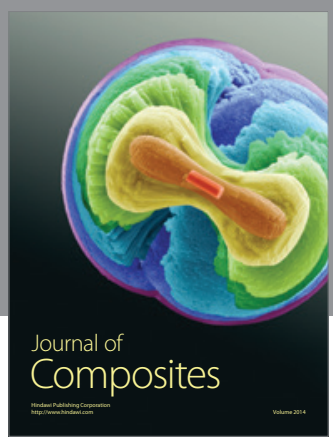
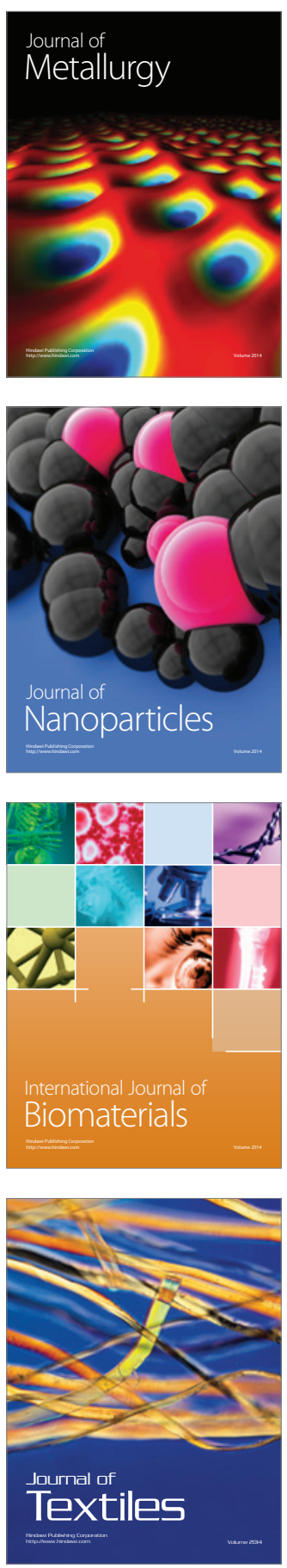